\title{
Ontology-Based Personalized Cognitive Behavioural Plans for Patients with Mild Depression
}

\author{
Aditi NAIR ${ }^{\mathrm{a}, 1}$, Syed Sibte Raza ABIDI ${ }^{\mathrm{a}}$, William VAN WOENSEL ${ }^{\mathrm{a}}$ and Samina \\ ABIDI ${ }^{b}$ \\ ${ }^{a}$ NICHE Research Group, Faculty of Computer Science, Dalhousie University, Canada \\ ${ }^{\mathrm{b}}$ Medical Informatics Faculty of Medicine, Dalhousie University, Canada
}

\begin{abstract}
Cognitive Behavioural Therapy (CBT) is an action-oriented psychotherapy that combines cognitive and behavioural techniques for psychosocial treatment for depression, and is considered by many to be the golden standard in psychotherapy. More recently, computerized CBT (CCBT) has been deployed to help increase availability and access to this evidence-based therapy. In this vein, a CBT ontology, as a shared common understanding of the domain, can facilitate the aggregation, verification, and operationalization of computerized CBT knowledge. Moreover, as opposed to black-box applications, ontology-enabled systems allow recommended, evidence-based treatment interventions to be traced back to the corresponding psychological concepts. We used a Knowledge Management approach to synthesize and computerize CBT knowledge from multiple sources into a CBT ontology, which allows generating personalized action plans for treating mild depression, using the Web Ontology Language (OWL) and Semantic Web Rule Language (SWRL). We performed a formative evaluation of the CBT ontology in terms of its completeness, consistency, and conciseness.
\end{abstract}

Keywords. Action Plans, Cognitive Behavioural Therapy, Knowledge Management, Mild Depression, Ontology, Semantic Web Rule Language

\section{Introduction}

Cognitive Behavioural Therapy (CBT) is a well-known psychosocial treatment for depression, enabling patients to cope with problems in cognition and behaviour [1]. Its effectiveness has been proven in a range of studies, and it is currently considered to be the gold standard in psychotherapy [2]. CBT relies on two main techniques: Guided Discovery (GD), which encourages patients to target dysfunctional thinking; and Behavioural Activation (BA), which encourages patients to engage in activities that increase one's mastery, i.e., perceived ability to perform the activity [1].

Computerized CBT (CCBT), with or without therapist support, has been widely deployed to improve access and availability of CBT, and several studies have shown its effectiveness in reducing anxiety and depression symptoms [3]. Nevertheless, the lack of consistency with evidence-based principles of CBT has resulted in questions about the safety and utility of many CBT apps [4]. We developed a biomedical CBT ontology that

\footnotetext{
${ }^{1}$ Corresponding Author, Aditi NAIR ; E-mail: aditi.nair@dal.ca.
} 
directly maps primary, evidence-based CBT principles into annotated concepts, relations, and axioms. In doing so, the CBT ontology ensures adherence to core ingredients of CBT, supports verification of its computerized CBT knowledge, and allows for evidence-based recommendations of treatment interventions.

Currently, our CBT ontology focuses on the computerization of (a) GD to allow patients to identify negative thought processes, which are associated with barriers that inhibit them from achieving a treatment goal; and (b) BA to promote activities that elicit feelings of mastery in a patient. The CBT ontology was created using the Web Ontology Language (OWL) and Semantic Web Rule Language (SWRL). Using a logical reasoner and patient profile instances, the ontology can be utilized to generate personalized action plans in line with GD and BA to reduce symptoms of mild depression.

\section{Constructing CBT Ontology}

To address the lack of soundness of CCBT solutions with regards to core CBT principles, we adopted a knowledge-modelling approach for constructing an OWL ontology. This ontology can be utilized as a common understanding of the CBT domain, supports verification of the underlying CBT principles, as well as checking for logical consistency and completeness. The knowledge-based solution approach is divided into three stages:

\subsection{Content Gathering}

We gathered relevant knowledge on CBT and its core concepts, including adapted treatment goals and the respective action plans for each goal, from Battle et al. [5]. Further, the knowledge model includes concepts from BDI-II [1] as barriers to treatment goals. We have incorporated the Decisional Balance Exercise (DBE) that lists the pros and cons of an action plan for a treatment goal [6]. We elaborate on these concepts below.

\subsection{Conceptual and Workflow Modelling}

Figure 1 summarizes the conceptualization of the CBT ontology. A Treatment Goal is a concrete long-term goal that a patient wants to achieve with the CBT treatment; such as improving social relationships, finding a job, or improving physical health. Action Plans $(A P)$ support a patient in attaining such a specific treatment goal. In particular, an AP aims to improve the patient's perceived ability at completing relevant, rewarding activities; such as running for 15 minutes every day (physical health), asking others for help, or showing physical affection (social relationships). A patient's perceived ability at these activities is represented by their Self-Efficacy Value (SEV). For an AP to be successful in improving a patient's self-efficacy, it is important that it is achievable; as failure at an AP may decrease one's self-efficacy. Hence, a Decisional Balance Exercise $(D B E)$ is incorporated to encourage realistic thinking, by listing pros and cons of an AP.

A Barrier stands for a psychological, logistical, or illness-related barrier that stops the patient from achieving their Treatment Goal. Such barriers may include feelings of sadness, guilt, failure, loss of interest, difficulty concentrating, and so on. Barriers are often caused by cognitive distortions, called Negative Thought Processes (NTP), including all-or-nothing thinking (e.g., if I'm not a success, I'm a failure), mind reading (i.e., assuming negative thoughts in others), mental filters (i.e., focusing solely on negative aspects), and so on. 


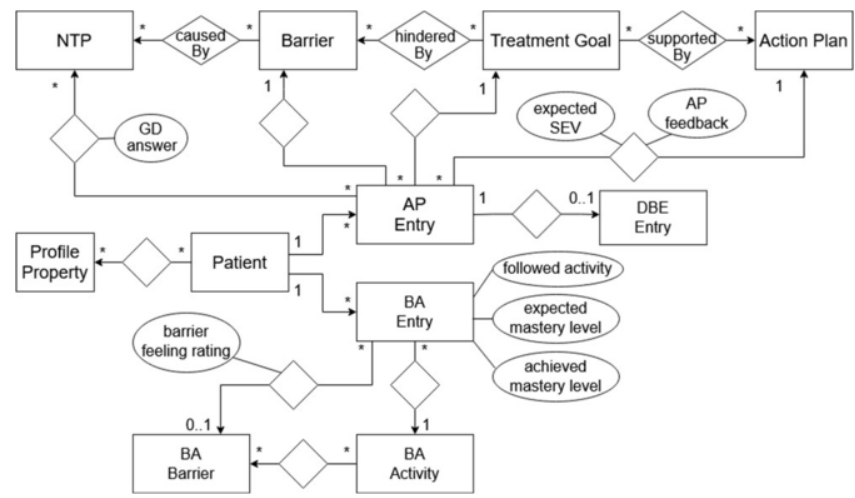

Figure 1. Summary of knowledge model conceptualization.

Our CBT ontology focuses on two main CBT techniques for psychosocial treatment. Guided Discovery (GD) aims to tackle cognitive dysfunctions (i.e., NTP) by posing questions such as: "What is an alternative way of viewing this situation?", "What is the worst that could happen, and how could you cope if it did happen?", and so on. Behavioral Activation (BA) tackles the inactivity of patients as this may lead to a vicious circle of increased negative feelings [1]. BA provides opportunities to gain a sense of mastery or pleasure by recommending short-term, rewarding activities; such as going on a picnic, watching a movie, and going out to dinner with friends. To that end, BA encourages individuals to perform activities, making note of (a) whether the patient followed the suggested activity, and (b) the expected and achieved levels of mastery.

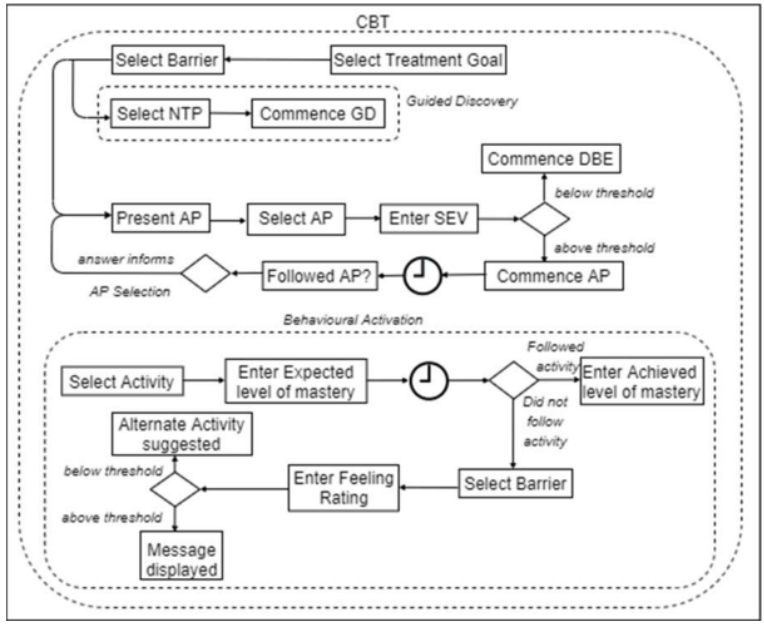

Figure 2. Computerized CBT workflow.

Figure 2 shows the computerized CBT workflow in line with GD and BA. We shortly summarize this workflow below.

A patient starts with selecting a long-term treatment goal, and a perceived barrier that stops them from attaining that goal. Subsequently, the following will take place:

(a) The patient may select NTP's associated with the barrier, and GD commences;

(b) Based on the treatment goal, barrier and the patient's profile, a set of personalized action plans are presented. The patient selects an action plan and enters an expected SEV; if it lies below a threshold, a $D B E$ will commence and list the pros and cons of the AP. 
Else, the AP will commence directly. After a given time (e.g., 1 week), the patient enters their feedback, indicating whether they were successful or not. Subsequently, other AP will be suggested, or the patient is recommended to try the same AP again;

(c) The patient selects an activity they would like to do once a week for BA, with a duration of less than 60 minutes or greater, depending on their preference. The patient rates their expected level of mastery and at the end of the week, if the patient did not follow the activity, they select a barrier that stopped them from doing so and enter a Barrier Feeling Rating; if it lies below a certain threshold, an alternate activity is suggested; whereas if it lies above the threshold, an appropriate message is displayed to the patient. If the patient followed the activity, then they are asked to enter the achieved level of mastery. This is recorded to monitor the patient's progress and maintain a record of activities that were helpful in reducing their depressive symptoms.

\subsection{Ontology Engineering}

We used Protege version 5.5 to develop the CBT ontology, which can be found online [7]. We modeled the concepts and relationships in Figure 1 using OWL classes and properties. We developed a set of SWRL rules that implement the computerized CBT workflow from Figure 2. These SWRL rules recommend personalized action plans to the patient, depending on the selected treatment goal and relevant profile properties (e.g., time spent watching TV, living near shopping mall). Given a concrete instantiation of the CBT ontology, a DL-reasoner is used to execute the computerized CBT workflow.

\section{Evaluation}

We assessed the CBT ontology by a qualitative evaluation that checked the completeness and consistency of the model in three case studies, which were validated by a clinical psychologist who is also a CBT practitioner. Given the concrete instantiation of the three case studies in the CBT ontology, the clinical expert found that appropriate, personalized GD and BA interventions were issued, given the patient profiles and other relevant input. Table 1 lists example inputs, expected output, and the actual inferred output.

Table 1. Case Study.

\begin{tabular}{lll}
\hline \multicolumn{1}{c}{ Input } & \multicolumn{1}{c}{ Expected Output } & \multicolumn{1}{c}{ Inferred Output } \\
\hline $\begin{array}{l}\text { Student, single, living in apartment } \\
\begin{array}{l}\text { Treatment Goal: Improve number of } \\
\text { non-family social relationships. }\end{array}\end{array}$ & $\begin{array}{l}\text { List Action Plan options for } \\
\text { selected Treatment Goal }\end{array}$ & $\begin{array}{l}\text { - Ask others for help. } \\
\text { - Think twice before speaking. } \\
\text { - Use words such as "thank you", } \\
\text { "please" and "thank you". }\end{array}$ \\
$\begin{array}{l}\text { Negative Thought Process: } \\
\text { Mind reading }\end{array}$ & $\begin{array}{l}\text { List Guided Discovery } \\
\text { questions }\end{array}$ & $\begin{array}{l}\text { - If someone I loved had this thought } \\
\text { what would I tell them? }\end{array}$ \\
& & $\begin{array}{l}\text { - If someone I loved knew I was } \\
\text { thinking this, what evidence would } \\
\text { they point out that would suggest that } \\
\text { my thoughts are not } 100 \% \text { true? }\end{array}$
\end{tabular}

Selected Action Plan:

Ask others for help. 


\author{
$1^{\text {st }}$ Self Efficacy Value (SEV): 0 \\ $2^{\text {nd }}$ Self-Efficacy Value (SEV): 2 \\ Selected activity for $B A$ : Going out \\ to dinner. \\ Expected Level of Mastery: 5 \\ Followed BA activity? Yes. \\ Achieved Level of Mastery: 8
}

List DBE associated with selected action plan

Display message for $2^{\text {nd }} \mathrm{SEV}=2$

If BA activity was followed, display message.
Pro - Gives opportunity for success through assistance by others. Con - Negative impact on your confidence.

Message: "Go ahead with your action plan."

Message: "Thank you for trying this activity! You can try the same activity or pick another for the next week."

\section{Discussion and Conclusion}

This paper presents a Semantic Web-based modelling approach for constructing a knowledge model for the self-management of CBT. The current lack of consistency of m-health CBT apps with theoretical principles of CBT motivated us to develop this CBT knowledge model [8]. The knowledge model is represented in terms of a CBT ontology where a logical reasoner, together with concrete patient data, can be used to generate personalized, effective action plans for treating mild depression in a specific patient; based on the relevant profile attributes and the selected treatment goal. Kumar et al. [8] conducted an analysis of m-health apps and found that the lack of focus on identifying barriers to treatment led to patient withdrawal [8]. This motivated us to create a knowledge model wherein barriers to treatment goals are instantiated.

A limitation of our approach is in the limited number of treatment goals modelled; if expanded upon in the future, such new goals would require discussions with domain experts, such as therapists. In the future, we plan to incorporate other components of CBT, namely, relaxation training [2] by adding suggestions for relaxation activities and monitoring. Furthermore, the model could suggest action plans to the user based not only on current SEV but also records of SEV from previous monitoring instances.

\section{References}

[1] Beck JS. Cognitive Behavior Therapy, Second Edition: Basics and Beyond. The Guilford Press; July 2011.

[2] David D, Cristea I, Hofmann SG. Why Cognitive Behavioral Therapy Is the Current Gold Standard of Psychotherapy. Front Psychiatry. 2018 Jan 29;9:4.

[3] Adelman CB, Panza KE, Bartley CA, Bontempo A, Bloch MH. A meta-analysis of computerized cognitive-behavioral therapy for the treatment of DSM-5 anxiety disorders. J Clin Psychiatry. 2014 Jul; 75(7):e695-704.

[4] Huguet A, Rao S, McGrath PJ, Wozney L, Wheaton M, Conrod J, Rozario S. A Systematic Review of Cognitive Behavioral Therapy and Behavioral Activation Apps for Depression. PLoS One. 2016 May 2;11(5): $\mathrm{e} 0154248$.

[5] Battle CL, Uebelacker L, Friedman MA, Cardemil EV, Beevers CG, Miller IW. Treatment goals of depressed outpatients: a qualitative investigation of goals identified by participants in a depression treatment trial. J Psychiatr Pract. 2010 Nov;16(6):425-30.

[6] The Ottawa Hospital Research Institute. Patient decision aids. Available at: https://decisionaid.ohri.ca/. Accessed 2020.

[7] GitHub. Available at: https://github.com/william-vw/CBTOntology. Accessed 2020.

[8] Kumar S, Mehrotra S. Free mobile apps on depression for Indian users: A brief overview and critique. Asian J Psychiatr. 2017 Aug;28:124-130. 